Barry Bloom (a Howard Hughes Investigator at the Albert Einstein College of Medicine) and Roy Widdus (Coordinator of the Children's Vaccine Initiative) describe their vision of global implementation of vaccines, highlighting what has already been achieved and, perhaps more importantly, what remains to be done. They also call for further efforts to develop the broad collaborations required to achieve a new vision for global vaccine use.

\title{
Vaccine visions and their global impact
}

Shortly after the assassination of Prime Minister Indira Gandhi in 1984, Jim Grant, Administrator of UNICEF and Hafdan Mahler, Director-General of World Health Organization (WHO), flew to New Delhi on an extraordinary mission. They proposed to her surviving son, Rajiv, that instead of building a shrine or temple, he honor his mother by making a commitment to immunize all the children of India against poliomyelitis by the end of the century. On December 6 and 7, 1996, in two National Vaccine Days, 121 million Indian children were vaccinated against polio at 650,000 immunization posts by 2.6 million volunteers. In one of the most staggering achievements in the history of public health, Gandhi's commitment was honored, and it continues. This was the result of vision and a remarkable concerted organizational effort by the government and the WHO Expanded Programme for Immunization (EPI), the provision of polio vaccine by Rotary International and UNICEF, foreign assistance from USAID, UK, Denmark, Korea and other overseas development agencies and the millions of volunteers.

It was possible not least because of the success of the first great vision for immunization in our time, the Global Smallpox Eradication campaign, which eliminated, for the first time in history, a dread disease from the face of the earth. That achievement was undertaken despite the general skepticism of public health officials, scientists and politicians at the time and showed that vaccines could effectively be delivered to poor people in poor countries-the second great vision. In 1974, 5\% of the world's children were immunized against childhood diseases. But by the mid-1990s, more than $90 \%$ of the world's children had received BCG vaccine for tuberculosis, and $75-85 \%$ had received vaccines for diphtheria, pertussis, tetanus, measles and mumps. The power of this vision of global vaccination has been enormous-in Salvador and in the Sudan, civil wars were stopped for Days of Tranquility to permit their children to be vaccinated. The WHO EPI programme saves 3 million children's lives each year and billions of dollars in medical costs. Proving even to economists that vaccines are among the most cost-effective interventions available to prevent death and disease ${ }^{1}$. Yet there is a new cohort of children born each year, and the vision is hard to sus- tain because, as Lewis Thomas noted, we tend to take for granted the technologies that work best ${ }^{2}$.

The third great vision-the eradication of paralytic poliomyelitis-is on the way to being achieved. Although the scientific problems of creating safe and effective polio vaccines were largely overcome by the 1960 s, to have global impact, political problems must also be overcome. Few countries want their infectious diseases to be publicly reported, and Ciro de Quadros of the Pan American Health Organization came up with a brilliantly imaginative solution to a delicate problem. Rather than identifying cases of paralytic poliomyelitis, he proposed symptomatic surveillance of acute flaccid paralysis, which is hardly a household word or politically explosive syndrome ${ }^{3}$. Thus from over 2000 cases of acute flaccid paralysis detected each year in the Western Hemisphere not a single case of paralytic poliomyelitis due to wild-type poliovirus has been reported in any of the 34 participating countries. It is expected that early in the next century, polio will become the second disease to be eliminated from the face of the earth by immunization. The introduction of many other childhood vaccines has produced a staggering reduction in death and disease (Fig.). For example, in the US. within a period of $5-10$ years after the introduction of vaccines, polio, diphtheria, neonatal tetanus, measles and rubella were all but eliminated (see Fauci, page 491). And childhood immunization is one of the most cost-effective medical interventions (Table 1). The U.S. Centers for Disease Control (CDC) estimates that for every dollar invested in immunization, between $\$ 2$ and $\$ 29$ are saved. The entire cost of the Global Smallpox Eradication program, about $\$ 32 \mathrm{~m}$, is returned every 20 days in not having to vaccinate travelers.

\section{The unfinished agenda}

Yet, infectious diseases, not cardiovascular disease, cancer or injuries, remain the largest cause of death and disability in the world, with an annual death toll of 17 million $^{4}$ (Table 2). Infectious diseases are not equitably distributed among countries of the world. They are responsible for $48 \%$ of the burden of premature death and disability in developing countries, and out of the eight leading causes of death and disability in devel- 


\begin{tabular}{lcc}
\hline Table 1 & $\begin{array}{c}\text { Cost effectiveness of childhood vaccines in the United } \\
\text { States }\end{array}$ \\
\hline & $\begin{array}{c}\text { (Savings per dollar invested) } \\
\text { Direct medical } \\
\text { Vaccine }\end{array}$ & $\begin{array}{c}\text { Direct and indirect } \\
\text { savings }\end{array}$ \\
savings \\
DTP & $\$ 6.0$ & $\$ 29.1$ \\
MMR & $\$ 15.3$ & $\$ 21.3$ \\
OPV & $\$ 3.4$ & $\$ 6.1$ \\
Integrated Schedule & $\$ 7.4$ & $\$ 25.5$ \\
(DTP, MMR, OPV combined) & & \\
H. influenza type b (Hib) & $\$ 1.4$ & $\$ 2.2$ \\
Hepatitis B (perinatal/infant) & $\$ 0.5$ & $\$ 2.0$ \\
Varicella & $\$ 0.9$ & $\$ 5.4$
\end{tabular}

Data from National Immunization Program, Centers for Disease Control and Prevention.

oping countries in 1990, six were directly or indirectly attributable to infectious diseases. Worldwide, four million children die of acute respiratory diseases and another 3 million from diarrheal diseases each year (Table 3 ). There are 8 million new cases and 2.5 million deaths from tuberculosis annually and 300-500 million cases and 2 million deaths due to malaria. UNAIDS estimates that 30.6 million people are currently infected with HIV and that this year, 2-3 million will die from AIDS. The numbers are steadily mounting-on the order of $40 \mathrm{mil}-$ lion people will die from AIDS in the next decade.

Although it has already been shown that in industrialized countries immunization can dramatically reduce hepatitis and hepatocellular carcinoma caused by HBV, and childhood meningitis caused by Haemophilus influenzae $b$ (Hib)

(US National Immunization Program, Centers for Disease Control and Prevention) relatively few developing countries have introduced these vaccines into their national vaccine programs. It will probably be necessary to demonstrate by local trials that these vaccines are cost-effective in their populations. This is now being undertaken for Hib in a collaboration between industry, WHO's Global Programme on Vaccines and the new International Vaccine Institute (IVI) in the Republic of Korea (see Shin, page 503). Recent efforts toward more effective prevention of measles, the single largest viral cause of death in children in developing countries, have proven successful in the Americas and are receiving greater emphasis in other parts of the world, including Africa. One can anticipate a major thrust to initiate a global campaign to eliminate measles, once polio is vanquished.

In the medium term, the latest vaccine submitted for licensure is a vaccine against rotavirus diarrhea, which kills 6-800,000 children each year. Other rotavirus candidate vaccines will follow (see Sansonetti, page 499) and the most effective should be put into wide use. New vaccines developed against Streptococcus pneumoniae (responsible for billions of cases of middle ear infections and 1.2 million childhood deaths from pneumonia worldwide), meningococcus $A$ and $C$ (which cause epidemic meningitis) and respiratory syncytial virus (also a major cause of death and illness from respiratory illness) need to be evaluated in clinical trials. Similarly, new vaccines against the major diarrheal diseases-typhoid fever, cholera, bacterial dysentery (shigellosis) and enteropathogenic $E$. coli-need to be evaluated in clinical trials in disease endemic areas.

Despite these developments, there remains an urgent need to develop new or improved vaccines against some of the more complex infectious diseases (Table 3). Parasitic diseases impose greater morbidity worldwide than viral or bacterial diseases, yet not a single vaccine is in use against any human parasite. Each year, malaria kills about 2 million people, mostly in Africa (see Miller \& Hoffman, page 520). It is hoped that the Multinational Initiative in Malaria will give some impetus to that effort ${ }^{5}$. And there are other important parasitic diseases for which vaccines could be of enormous value-schistosomiasis, which causes by worms that damage the liver or the bladder predisposing to cancer; leishmania, that cause disfiguring localized and sometimes fatal systemic disease; Entamoeba the cause of amoebic dysentery; and leishmaniasis, which causes causes Chagas disease in Latin America, caused by trypanosomes.

Sexually transmitted diseases (STD), including HIV/AIDS are out of control in many developing countries. In fact, HIV/AIDS is one of two health conditions worldwide with increasing mortality (the other is tobacco-related deaths). Although major efforts are underway to develop vaccines against AIDS, this is likely to be years away (see Burton \& Moore, page 495, and Heilman \& Baltimore, page 532 ). The most prevalent STD is caused by chlamydia species, which are a major predisposing cause of transmission of HIV $^{6}$. Trachoma, caused by a related species, remains a major cause of blindness in children. Neisseria gonorrhoeae and Treponema pallidum (the agent that causes syphilis) are also STD pathogens for which vaccines are urgently needed.

\section{The coming epidemic}

In their classic study of the causes of death and disability from disease, Murray and Lopez ${ }^{4}$ projected secular trends in an attempt to develop a picture of the global burden of disease in the year 2020 (Table 2). If one makes the assumptions that new global infectious diseases that increase the burden of infectious diseases significantly will not arise, and particularly that emergent drug resistance does not reverse the present downward trends of most infectious diseases, the major burdens of disease in the year 2020 will be cardiovascular disease, neuropsychiatric

Table 2 Burden of disease in industrialized and developing countries, 1990

\begin{tabular}{|c|c|c|c|c|c|c|}
\hline \multirow[t]{3}{*}{ Condition } & \multirow{2}{*}{\multicolumn{2}{|c|}{$\begin{array}{c}\text { Deaths (millions) } \\
1990\end{array}$}} & \multicolumn{4}{|c|}{$\%$ DALYS } \\
\hline & & & \multicolumn{2}{|c|}{1990} & \multicolumn{2}{|c|}{2020} \\
\hline & $\begin{array}{l}\text { Developing } \\
\text { countries }\end{array}$ & $\begin{array}{l}\text { EME/FSE } \\
\text { countries }\end{array}$ & $\begin{array}{l}\text { Developing } \\
\text { countries }\end{array}$ & $\begin{array}{l}\mathrm{EME} / \mathrm{FSE} \\
\text { countries }\end{array}$ & $\begin{array}{l}\text { Developing } \\
\text { countries }\end{array}$ & $\begin{array}{l}\mathrm{EME} / \mathrm{FSE} \\
\text { countries }\end{array}$ \\
\hline $\begin{array}{l}\text { Communicable, maternal } \\
\text { and perinatal }\end{array}$ & 16.6 & 0.67 & 48.7 & 7.8 & 22.2 & 4.3 \\
\hline Cardiovascular & 9.1 & 5.2 & 8.2 & 20.4 & 13.8 & 19.4 \\
\hline Neoplastic & 3.6 & 2.4 & 4.0 & 13.7 & 9.0 & 16.8 \\
\hline Injuries & 4.3 & 0.83 & 15.2 & 14.5 & 21.1 & 10.1 \\
\hline Total & 39.6 & 10.9 & 1,220 & 161 & $1,228 \mathrm{~m}$ & $161 \mathrm{~m}$ \\
\hline
\end{tabular}

DALYS = Disability Adjusted Life Years, a measure of premature death and disability.

$\mathrm{EME}=$ Established Market Economies; $\mathrm{FSE}=$ Former Soviet Economies. (Adapted from ref. 4.)

Other conditions not included in the table contribute to the total deaths and and DALYS. 
disease, injuries and cancers (see Pardoll, page 525). This new epidemiologic transition has been referred to as the coming epidemic' of chronic, non-communicable diseases. Nevertheless, of the top 12 causes of death and disability projected for 2020 , seven will still be classical infectious diseases, including HIV and TB.

It is increasingly recognized that intriguing associations exist between certain chronic non-communicable diseases and infectious pathogens ${ }^{7}$. Overcoming years of skepticism, it is now clear that Helicobacter pylori is the principal cause of peptic ulcer disease, and a major predisposing risk factor for stomach cancer ${ }^{8}$. And rheumatic fever, initiated by Group A streptococcus infection, and chronic Lyme Disease have taught us that devastating autoimmune sequelae can be initiated by infectious agents. There are also associations between Chlamydia pneumoniae and coronary artery disease $^{9}$, and herpesviruses, particularly cytomegalovirus, have been associated with coronary artery restenosis in humans ${ }^{10}$. Mycobacterial DNA has been associated with granulomatous lesions in Crohn's disease, a chronic inflammatory bowel disease ${ }^{11}$ and human herpes virus 6 (HHV-6) has recently been associated with multiple sclerosis ${ }^{12}$, HHV-8 with human Kaposi's sarcoma ${ }^{13}$ and arteritis in animal models ${ }^{14}$. A variety of diseases of unknown etiology, including Whipple's disease, Wegener's granulomatosis and sarcoidosis, have pathological features or DNA 'footprints' associating them with chronic infections, the etiologic agents for which have not been isolated or cultured, and perhaps may never be. In some cases, the associations are tenuous and inconclusive, and may be explained as merely passenger effects. In others, such as Whipple's disease, the association is causal, even though the agent defined by PCR, Tropheryma whippelii, has not yet been cultured ${ }^{15}$. But even if only some of these associations are found to be causal, major opportunities will be presented for developing a new line of vaccines against pathogens contributing to the so-called non-communicable diseases that contribute increasingly to the burden of disease.

\section{IMAGE UNAVAILABLE FOR COPYRIGHT REASONS}

Finally there are two more areas in which vaccines could become major therapeutic and prophylactic interventions. The first is cancer. Human papillomavirus is highly associated with cervical cancer, the second leading cause of death in women from cancer in developing countries ${ }^{16}$. As described by Drew Pardoll on page 525 of this issue, tumor-associated antigens have been identified in several human tumors and represent promising targets for new vaccines. Autoimmune and immunological diseases represent the other new vaccine frontier (see Cookson \& Moffatt, page 500). When inappropriate immune responses are raised against body tissues or environmental antigens, it may be possible to immunize against the autoantigens or the $\mathrm{B}$ and $\mathrm{T}$ cell receptors themselves, to modulate the responses and ameliorate the diseases. One can imagine that immunological interventions could have significant impact on myasthenia gravis, lupus erythematosis, rheumatoid arthritis, multiple sclerosis, allergies and asthma, for example. And in the more distant future, when the human genome is sequenced and it is relatively easy to correctly define genetic risks for certain diseases, prophylactic immunization against certain kinds of cancer and autoimmune disease may be conceivable.

\section{Challenges to the vision-science}

Whereas the vision is quite clear, achieving it will not be straightfoward. The easy vaccines have been made. Pickling the remaining pathogens, even if they can be grown in culture, is unlikely to yield effective vaccines against many of the diseases adumbrated here. Many scientific challenges stand between us and the new vaccines.

Conformational epitopes. Whereas $\mathrm{T}$ cells recognize small linear peptides in the context of $\mathrm{MHC}$ molecules, antibodies that neutralize viruses generally recognize antigenic determinants that are created by the three-dimensional conformation of the protein or glycoprotein antigens on the pathogen. Isolated or recombinant proteins, although antigenic, as in the case of the poliovirus capsid protein, often fail to elicit neutralizing antibodies essential for protection. One of the major limitations of the current HIV/AIDS subunit vaccine candidates is their inability to present the neutralizing epitopes in a conformationally faithful manner that will result in antibodies capable of neutralizing field isolates. Polymorphisms. Some of the major pathogens for which vaccines are needed, particularly rapidly mutating RNA viruses such as HIV and influenza viruses, and parasites, for example, plasmodia that cause malaria or trypanosomes that cause sleeping sickness, undergo a rapid mutation and selection by immune responses for antigenic variants. Thus antibodies to many pathogens, and to a lesser extent, $\mathrm{T}$ cell responses, are often restricted to the initial immunizing strain or type. How we develop broad specificity vaccines that will reduce the selection capacity of escape mutants, and 
anticipate at least the major antigenic variants likely to emerge, remains unknown. The Age Barrier. In endemic countries many children are exposed to infectious pathogens very early in life, yet some existing vaccines are given later in life. For example, in the US we immunize against measles at 12-18 months so that the vaccine is not neutralized by maternal antibodies. However, in developing countries many children would die if the vaccine were withheld until that time. In some countries, measles vaccine is delivered as early as six months-an age at which the immunologic response is suboptimal but the compromise provides additional protection against the early onslaught of measles infection. And we know that rotavirus vaccines are more effective in countries where children are not infected with high exposure very early in life. One of the greatest challenges is vaccines against respiratory syncytial virus (RSV), that strikes children younger than two years of age. These realities create a compelling need for research on how to generate effective protective immune responses in the first months of life. Immunologic Memory. There is an unfortunate, but understandable, proclivity of immunologists to report the protective efficacy of their experimental vaccines at about ten days after the third booster shot, when it is maximal. However, for immunization against tuberculosis, HIV/AIDS and malaria, where exposure can occur from early age to anytime in life thereafter, it is essential that vaccines prime a long term immunologic memory. With memory, infection may occur but disease will be prevented or at least attenuated. In endemic circumstances, infection with the pathogen may even serve as a booster and stimulate accelerated protective responses. Although most long-term memory is carried by long-lived $\mathrm{T}$ cells, ideally both immunological memory and persisting effector responses, mediated by antibodies, $\mathrm{T}$ helper or cytotoxic $T$ cells, should be generated. Compartmentalization of Immune Responses. Not all immune responses to a pathogen are protective-some cause tissue damage and disease. Therefore, for each pathogen, one needs to understand the necessary and sufficient immune responses required to protect, while minimizing pathology. For some agents, particularly viruses, antibodies are needed to neutralize the infection. For many intracellular bacterial and protozoal pathogens, $\mathrm{T}$ cells producing lymphokines are needed, and, if as in experimental models of leishmaniasis in mice, the wrong constellation of lymphokines is produced, disease can be exacerbated. For many intracellular pathogens, cytotoxic $T$ cells are essential, and yet the conditions for present-

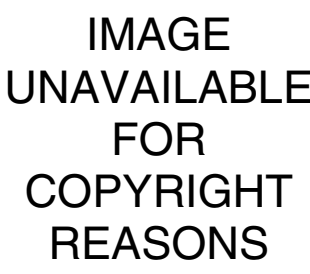
ing antigens to engender CTL, requiring presentation through $\mathrm{MHC}$ class I molecules, are different from those inducing $\mathrm{T}$ cells producing lymphokines. And for respiratory and sexually transmitted pathogens, systemic immune responses are often inadequate to block infection, and effective mucosal immune responses are required. Targeting vaccines to specific compartments of the immune system is yet another major scientific challenge.

\section{Operational challenges}

The pricing and scale of production of vaccines are critical both to their development and implementation (see Batson, page 487). The initial investments in research and trials are high, perhaps $\$ 250 \mathrm{~m}$ dollars per vaccine, and will only be forthcoming if there are prospects for reasonable future returns. In a sense, the traditional vaccines-available to developing countries through UNICEF at well below $\$ 1$ for a series-are too cheap. These prices are possible because of the steadily improved manufacturing efficiency over the 25-50 years they have been in production. For new vaccines, however, the manufacturing costs per dose will be much higher until sufficient experience makes it possible to increase production efficiency. Yet the expectation exists that the new vaccines will be as cheap as the old ones, and this thwarts development and discourages industry. Furthermore, the willingness of the public to pay for prevention is, regrettably, less than that for treatment. The consequences are predictable. For diseases afflicting poor people in poor countries (cholera, dysentery, pneumonia, malaria and, to an extent, even AIDS) the possibilities for financial returns on investments are small. Yet setting higher prices on 'orphan' vaccines for diseases of developing countries would mean they will never reach the patients most in need of them. Where new vaccines are needed in both developed and developing countries, a mechanism of tiered pricing is clearly needed. Industrialized countries must be responsive to the expectations of their populations that they will introduce new vaccines into use, even at initial, higher prices, that would preclude use in low-income countries. Tiered prices can make vaccines affordable for developing countries and provide economies of scale on production costs through sharing overheads over a larger production volume (see Saldarini, page 485). Tiered pricing allows marginal profits to be made on sales to poor countries in return for the advantages of higher production volume. However, even at price tiering of say $80 \%$ to the poorest countries, the cost of vaccination programs is still high as a fraction of per capita GNP in the poor countries, compared to industrialized countries. Issues of affordability, subsidy and price gauging become increasingly complex.

New vaccines and technologies are covered by patents, for which licensees pay royalties, usually as a percentage of the selling price. Royalties can aggregate to over $10 \%$ of the cost as in the case of Hepatitis B vaccine. One could contemplate the possibility of tiering of royalties by inventors and companies as well. Would the inventors in the scientific community and industry be willing to tier their royalties to assure greater use of their vaccines in poor developing countries?

Finally, for the most needed 'orphan' vaccines to prevent diseases predominantly in the $85 \%$ of the world's population that lives in developing countries, new mechanisms are needed to reduce the financial risks and costs to industry of developing vaccines which, however important to public health, are unlikely in 
the foreseeable future to have adequate financial markets.

A further operational challenge to vaccine implementation might be called Campaign Fatigue and Dislocation of Primary Health Services. The very success of National Immunization Days points to a major problem. Millions of people can be mobilized to wipe out polio over a period of $2-8$ days per year. In the process, other routine health programs may be disrupted for months to ensure the success of the campaigns, and the chance to attack other health problems is forgone. The problem lies not in polio eradication efforts per se, but rather in the difficulty in securing resources to strengthen routine vaccine delivery systems. How can the enthusiasm for immunization four days a year be converted to strengthening routine immunization through primary health care services, so that children that have any contact with the health care system can be immunized 365 days of the year? Without such infrastructure, as childhood deaths diminish, complacency is likely to increase and the old scourges, such as diphtheria in Russia in the mid-1990s, will return.

This infrastructure must reach beyond infants. Outbreaks of measles in the US this decade highlighted the necessity for immunizing high school children and adults. In the US, 60-70,000 adults die of influenza and pneumonia annually, a major portion of which could be prevented if there were an effective system to provide adult immunization (according to a US National Vaccine Advisory Committee 1994 report). As new vaccines are developed against HIV/AIDS, STDs and tuberculosis, for example, and perhaps against some chronic diseases, their impact will be dependent on our ability to develop systems and strategies for adolescent and adult immunization.

\section{A new vision for the future}

The Children's Vaccine Initiative Strategic Plan ${ }^{17}$ estimates that wider use of currently licensed but underused vaccines, including measles, HBV, Hib and rubella, and the introduction of vaccines currently in late stages of development, such as rotavirus and pneumococcal vaccines, could save up to 6 million lives annually within the next decade. If some of the more ambitious actions proposed were implemented, 12 million lives could be saved. It took a decade after $\mathrm{HBV}$ vaccines were licensed for them to be introduced into public health programs in developing countries and this process has barely begun in the poorest countries of sub-Saharan Africa. Over 800,000 potentially fatal infections still occur unnecessarily each year. And it has taken more than five years for Hib vaccines to enter the immunization programs of even the richer developing countries. The rate of progress remains slow despite evidence that the disease kills over 500,000 children each year. This patchwork approach to coverage is inadequate, inequitable and inappropriate.

Let us not forget that most measles and rubella cases in the US are imported, just as smallpox and polio used to be. Richer countries remain vulnerable to infectious diseases that are allowed to rage in developing countries. A commitment is needed to bring all new vaccines, as they are developed and shown to be effective, rapidly into public health use everywhere.

We ask, what greater public responsibility has any nation than to protect the lives of its people, its children, and its future? Most countries of the world can afford to allocate their own national resources to assure that their children get the WHO recommended childhood vaccines and except in the countries with the direst of needs, that is not where foreign assistance should be directed. Where possible, international assistance should be used to accelerate the development and introduction of new vaccines. But just as we know that better health is linked to a country's economic development ${ }^{2}$, it will not be possible to do more with inadequate resources. For the new benefits that new vaccines will bring, new resources must be allocated. An increase in national, bilateral and multilateral public sector support is needed to solve the many scientific problems of developing new vaccines and to upgrade the global infrastructure for testing, evaluating and delivering vaccines. And a new kind of public-private collaboration will be needed to ensure that the high risks and costs of vaccine development will not prevent the development of those needed to protect the lives of people in the poorest countries.

The diseases are there. The global delivery system is almost there. The science is getting there. What is lacking is a shared commitment to this vision of a future in which all countries benefit from the protection that vaccines can bring. As the Book of Proverbs teaches, 'Where there is no vision, the people perish'.

\section{Acknowledgments}

We are grateful to David Relman (Stanford Medical School) for providing his review of infectious agents and chronic diseases prior to publication, to Maureen Birmingham (WHO Global Programme for Vaccines) for information on the immunization compaigns in India and China, to Susan Raymond (NY Academy of Sciences) for the reference for Fig. 1 and to Tore Godal (WHO Tropical Diseases Research Prog.) for information on the burden of infectious diseases.

1. World Bank. World Development Report 1993: Investing in Health. NY. Oxford Univ. Press. (1993)

2. Thomas, L. The Lives of the Cell: Notes of a Biology Watcher. Penguin. pp. 153 (1995).

3. de Quadros, C.E. et al. Eradication of poliomyelitis. Progress for the Americas. /. Ped. Infect. Dis. 10, 222-229 (1991).

4. Murray, C.J.L \& Lopez, A. The Global Burden of Disease and Injury. Vol 1. Harvard Univ. (1996).

5. Editorial. Two cheers for the multilateral malaria initiative. Nature 388, 211 (1997).

6. Plummer, F.A. et al. Cofactors in male-female sexual transmission of human immunodeficiency virus type 1. J. Infect. Dis. 163, 233-239 (1991).

7. Fredricks, D.N. \& Relman, D.A. Infectious agents and the etiology of chronic idiopathic diseases. Curr. Clin. Topics Inf. Dis. (In the press).

8. Tytgat, G.N., Noach, L.A. \& Rauws, E.A. Helicobacter pylori infection and duodenal ulcer disease. Gastroenterol. Clin. North. Amer. 22, 127-139 (1993).

9. Saikku, P. et al. Chronic Chlamydia pneumoniae infection as a risk factor for coronary heart disease in the Helsinki Heart Study. Ann. Intern. Med. 116, 273-278 (1992)

10. Zhou, Y.F. et al. Association between prior cytomegalovirus infection and the risk of restenosis after coronary atherectomy. N. Engl. ). Med. 335, 624-630 (1996).

11. Sanderson, J.D., Moss, M.T., Tizard, M.L. \& Hermon, T.J. Mycobacterium paratuberculosis DNA in Crohn's disease tissue. Gut 33, 890-896 (1992).

12. Soldan, S.S. et al. Association of human herpes virus 6 (HHV-6) with multiple sclerosis: Increased IgM response to HHV-6 early antigen and detection of serum HHV-6 DNA. Nature Med. 3, 1394-1397 (1998).

13. Chang, $Y$ et al. Identification of herpesvirus-like sequences in AIDS-associated Kaposi's sarcoma. Science 266, 1865-1869 (1994).

14. Weck, K.E. et al. Murine $\gamma$-herpesvirus 68 causes severe large-vessel arteritis in mice lacking interferon-responsiveness; A new model for virus-induced vascular disease. Nature Med. 3, 1346-1354 (1997).

15. Relman, D.A., Schmidt, T.M., MacDermott, R.P. \& Falkow, S. Identification of the uncultured bacillus of Whipple's disease. N. Engl. J. Med. 327, 293-301 (1992).

16. Schiffman, M.H. New epidemiology of human papillomavirus infection and cervical neoplasia. J. Natl. Cancer Inst. 87, 1345-1347 (1995).

17. Children's Vaccine Initiative. Strategic Plan. WHO. Geneva (1998).

${ }^{1}$ Investigator, Howard Hughes Medical Institute and Weinstock Professor of Microbiology and Immunology at the Albert Einstein College of Medicine 1300 Morris Park Avenue

Bronx, New York, 10461, USA

${ }^{2}$ Coordinator, Secretariat

Children's Vaccine Initiative

20 Avenue Appia, 1211 Geneva 27, Switzerland 